\title{
Development and Validation of the Medical Affairs Pharmaceutical Physician Value (MAPPval) Instrument
}

\author{
Ravi Jandhyala ${ }^{1,2}$ (D) \\ Accepted: 30 November 2021 / Published online: 7 January 2022 \\ (c) The Author(s) 2022
}

\begin{abstract}
Background A pilot study conducted in 2020 suggested that Medical Affairs Pharmaceutical Physicians (MAPPs) may be inherently undervalued within the pharmaceutical industry and vulnerable to replacement by less qualified roles. There are currently no standardized metrics to measure MAPP performance, thus it is necessary to measure the value of MAPPs to employers and clarify the need for their specific skills.

Objectives The first aim of this study was to identify a list of indicators to produce an MAPP value measurement tool, and the second aim was to determine its discriminant validity by showing that the 'MAPPval instrument' differentiated the MAPP role from other internal stakeholders (regulatory affairs, market access, commercial, and patient advocacy) in terms of accountability for pharmaceutical company activities and level of engagement with external stakeholders.

Methods MAPPs were recruited using convenience sampling via professional networks and completed a qualitative online survey to identify a list of key role indicators using a consensus method known as the Jandhyala method. Responses were coded and scored, and aggregated responses were presented to participants in a Consensus Round. Participants rated their agreement with each item on a 5-point Likert scale, from strongly agree to strongly disagree. Indicators that reached a consensus index of $>50 \%(\mathrm{CI} \geq 0.51)$ were retained in the final MAPP performance instrument. Participants' retrospectively self-reported professional activities over a period of 12 months were used to validate the measure. A two-proportion $z$-test and Mann-Whitney tests were used to determine discriminant validity by showing whether the value of the MAPP role as defined by the instrument was significantly different from that of other internal stakeholders in terms of their accountability for and external stakeholder benefit from each MAPP activity.

Results In total, 11 MAPPs participated in the Jandhyala method, which generated 22 unique MAPP value indicators. Payor-targeted activities and journal publications had the two highest awareness indexes ( 1.00 and 0.98 , respectively). The retrospective study confirmed the MAPPval instrument's validity. MAPPs were the only internal stakeholder classified as accountable for at least one activity that benefited all four stakeholders. They were classified as accountable for activities that influenced significantly more external stakeholders than other internal stakeholders, even when activities influenced fewer than four external stakeholders. MAPPs were also accountable for significantly more activities recorded over the 12-month period than regulatory affairs, market access, commercial, and patient advocacy.

Conclusions This study generated and validated the first measure of MAPP value to pharmaceutical companies. MAPPs have unique value to pharmaceutical companies compared with other roles in terms of their accountability for activities that influence regulators, payors, prescribers, and patients. Through their accountability for pharmaceutical company activities and influence of external stakeholders, MAPPs play a key role in medicine adoption.
\end{abstract}

Ravi Jandhyala

ravi@medialis.co.uk

1 Medialis Ltd, 13 Horse Fair, Banbury, Oxford OX16 0AH, UK

2 Faculty of Life Science and Medicine, Centre for Pharmaceutical Medicine Research, Institute of Pharmaceutical Science, King's College University, Franklin Wilkins Building, 150 Stamford Street, London SE1 9NH, UK

\section{Introduction}

The role of Medical Affairs Pharmaceutical Physicians (MAPPs) within the pharmaceutical industry has not always been well understood [1]. Furthermore, the role has evolved in recent years, meaning that internal and external stakeholders may have different understandings of its activities [2]. Recent targeted research with a focus group of MAPPs 


\section{Key Points}

This study developed the first-ever unique Medical Affairs Pharmaceutical Physicians (MAPP) value measurement tool, the 'MAPPval instrument'.

MAPPs were the only internal stakeholder accountable for activities that benefited all four external stakeholders involved in medicine adoption.

The MAPPval instrument was validated by successfully discriminating between the value of MAPPs and other internal stakeholders to pharmaceutical companies.

highlighted a consistent lack of understanding among their colleagues regarding the value their role provides to the organization, and that this was driven by the lack of measurement metrics (Jandhyala R, MBBS, unpublished data, 2020). Elsewhere, extensive work by the International Federation of Associations of Pharmaceutical Physicians and Pharmaceutical Medicine and other leading experts [2, 3] has advocated for the role and defined its competencies, activities, and importance to drug development, adoption, and post-adoption and launch processes [4, 5]. They have been recognized as influencing engagements with internal and external stakeholders and thereby contribute extensively to multi-stakeholder involvement in medicine launch and adoption. Collaboration between stakeholders across the many broad roles in the pharmaceutical industry $[6,7]$ is required to optimize medicine prescribing and improve patients' health [8], therefore engagement with stakeholders external to pharmaceutical companies is vital to their success. MAPPs may be involved in research that identifies and communicates gaps between actual practice and evidence-based guidelines [9-14], improving patient outcomes and decreasing healthcare costs [15-17]. Additionally, MAPPs play an active role in the movement of medicines from pharma to regulator, payor and prescriber, facilitating engagements between the pharmaceutical companies and external stakeholders [7], and may hold greater weight with prescribers than those in non-medically qualified roles [18]. Finally, MAPPs have a responsibility for pharmacovigilance and must ensure the safety of medicines post-launch [6].

\subsection{Measuring the Value of the Medical Affairs Pharmaceutical Physician}

Due to the varied and technical role of MAPPs, measuring their performance within the pharmaceutical industry requires a multifaceted approach. Unlike the pharmaceutical sales representative, whose performance can be measured based on the number of products sold and the revenue generated [19], there are no standardized metrics for measuring the performance of MAPPs. The core competencies needed by MAPPs within the pharmaceutical industry have been established [20], but their performance and contribution have not been appropriately evaluated and measured to date. To achieve this, MAPP value must be theoretically conceptualized as a construct and operationalized at an empirical level. This process requires the generation of a definition of the construct and the identification of indicators that must be observed to measure the construct accurately.

\subsection{Study Aims}

The aims of this study were to (1) develop a simple MAPPval instrument by identifying a list of core indicators needed to measure MAPP value using the Jandhyala method [21]; and (2) validate the instrument by showing its ability to differentiate the MAPP role from that of other internal stakeholders in terms of the value it provides to pharmaceutical companies by answering the following research questions.

(a) Is the MAPP the only internal stakeholder who has been classified as accountable for at least one activity benefiting each of the four external stakeholders?

(b) Has the MAPP been classified as accountable for activities benefiting significantly more external stakeholders than each of the other internal stakeholders?

(c) Is the number of times the MAPP has been classified as 'consulted about', 'responsible for', or 'informed about' significantly greater than each of the other internal stakeholders?

\section{Methods}

\subsection{Participants and Recruitment}

A total of 11 MAPPs were recruited using convenience sampling via professional networks, and were invited to participate in the study between 9 and 23 June 2021. Of these, 11 participated in the Awareness Round and 10 participated in the Consensus Round, which took place between 31 May 2021 and 12 June 2021. To be included, participants had to have at least 2 years of medical affairs experience at a UK pharmaceutical company at the regional or global level. There was no geographic limitation for inclusion, but 10 participants were located in the UK at the time of this study. Participants were informed that taking part in the study was voluntary and were given information about how to withdraw. Written informed consent was obtained from all participants after providing information about the study and 
before the study commenced. Responses were anonymized and Consensus Round list items were not identifiable to particular participants. In accordance with international regulations, ethical approval for this study was granted by King's College London Research Ethics Committee (reference number: MRA-20/21-22441).

\subsection{Development of the MAPPval Instrument}

MAPPs were invited to complete a qualitative online survey about their role to identify a list of key indicators that defined their value to pharmaceutical companies using a consensus method known as the Jandhyala method [21]. The Jandhyala method uses a novel approach that is distinct from other consensus methods, such as the Delphi and modified-Delphi approaches, as it contains metrics at the awareness and consensus stages to provide a quantification of participants' awareness of, and agreement with, each list item generated [22]. It has been used to develop instruments similar to the MAPPval instrument [23, 24]. The online survey invited participants to provide at least 3 , and up to 50 , free text responses, each referring to one MAPP value indicator, in response to the following question: What are the performance metrics, or indicators, that an MAPP should be measured on? All responses were coded by two research analysts, with discrepancies settled by the author. Participants' survey responses received a score of one for each code they referred to. This comprised the awareness score, which showed how much knowledge each participant contributed.
Aggregated coded responses were then presented to participants in an anonymized online survey in the Consensus Round in which they were asked to rate their agreement with each item on a 5-point Likert scale, from strongly agree to strongly disagree. Indicators that reached a consensus index of $>50 \%(\mathrm{CI} \geq 0.51)$ were retained in the final MAPPval instrument.

\subsection{Validation of the MAPPval Instrument}

After the Consensus Round, the discriminant validity of the MAPPval instrument was assessed by implementing the MAPPval instrument in a retrospective study of MAPP job activities over a period of 12 months, covering 1 July 2020 to 30 June 2021. A total of 12 MAPP activities (Table 1), four external stakeholders, and five levels of accountability were identified as central to MAPP value from the Jandhyala method-generated consensus (Table 2). These were applied to MAPPs' self-reported job activities over the 12-month period to determine whether the relative value of MAPPs to pharmaceutical companies varied significantly compared with regulatory affairs, market access, commercial, and patient advocacy stakeholders. A discriminative scale was developed from the MAPPval instrument, which defined value according to whether internal stakeholders were accountable for, consulted about, responsible for, informed about, or not involved in (ACRIN) each activity. The ACRIN scale indicated the level of value of each internal stakeholder from 'accountable' (the highest level of involvement) to 'not

Table 1 Medical affairs pharmaceutical physicians activity types extracted from the 'MAPPval instrument'

\begin{tabular}{|c|c|}
\hline Activity type & Description \\
\hline Plan & $\begin{array}{l}\text { A documented course of action for conducting a series of value-adding activities for a set period } \\
\text { of time }\end{array}$ \\
\hline Submissions & $\begin{array}{l}\text { A piece of work, which leaves the company, conducted for the purpose of influencing an external } \\
\text { stakeholder with a documented argument supported with evidence }\end{array}$ \\
\hline Protocol (IIS/CSS) & $\begin{array}{l}\text { Participating in the development, review, or progression of a protocol for a clinical study irre- } \\
\text { spective of sponsorship }\end{array}$ \\
\hline Clinical study (report) & Participating in the progression of a clinical study to the point of completion \\
\hline Journal publication & $\begin{array}{l}\text { Participation in the design, development, and progression of an academic manuscript that culmi- } \\
\text { nates in its publication in a peer-reviewed, indexed journal }\end{array}$ \\
\hline Congress poster & $\begin{array}{l}\text { Participation in the design, development, progression of an academic poster culminating in its } \\
\text { acceptance in an academic congress }\end{array}$ \\
\hline Material certification or examination & Certification or medical examination of materials \\
\hline Meetings: One-to-one & A personal meeting with a single external stakeholder (face-to-face, online, or teleconference) \\
\hline Meetings: One-to-many & $\begin{array}{l}\text { A personal meeting with a group of members of a stakeholder (face-to-face, online, teleconfer- } \\
\text { ence) }\end{array}$ \\
\hline Meetings: Insight-generating advisory board & Participation in an 'advisory board meeting' \\
\hline Meetings: Congress & Participation in a meeting at an academic congress \\
\hline Meetings: Training & $\begin{array}{l}\text { Participation in training internal or external stakeholders to a required level of competency or } \\
\text { knowledge }\end{array}$ \\
\hline
\end{tabular}

CSS company-sponsored study, IIS investigator-initiated study 
Table 2 'MAPPval instrument'

\begin{tabular}{|c|c|c|c|c|c|c|c|}
\hline \multirow[b]{2}{*}{$\begin{array}{l}\text { Long Title of activity } \\
\text { (Free text) }\end{array}$} & \multirow[b]{2}{*}{ Activity type } & \multirow[b]{2}{*}{$\begin{array}{l}\text { External Stakeholder } \\
\text { Audience }\end{array}$} & \multicolumn{5}{|c|}{ Level of value in activity } \\
\hline & & & $\grave{\vdots}$ & 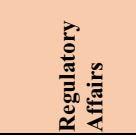 & 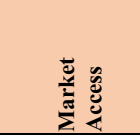 & 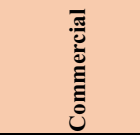 & 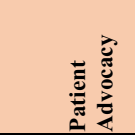 \\
\hline & Plan & Regulator & Accountable & Accountable & Accountable & Accountable & Accountable \\
\hline & Submissions & Payor & Consulted & Consulted & Consulted & Consulted & Consulted \\
\hline & Protocol (IIS/ CSS) & Prescriber & Responsible & Responsible & Responsible & Responsible & Responsible \\
\hline & Clinical Study (Report) & Patient & Informed & Informed & Informed & Informed & Informed \\
\hline & Journal Publication & & Not Involved & Not Involved & Not Involved & Not Involved & Not Involved \\
\hline & Congress Poster & & & & & & \\
\hline & $\begin{array}{l}\text { Material certification or } \\
\text { examination }\end{array}$ & & & & & & \\
\hline & Meetings: 1 to 1 & & & & & & \\
\hline & Meetings: 1 to many & & & & & & \\
\hline & $\begin{array}{l}\text { Meetings: Insights } \\
\text { generating Advisory board }\end{array}$ & & & & & & \\
\hline & Meetings: Congress & & & & & & \\
\hline & Meetings: Training & & & & & & \\
\hline
\end{tabular}

Italicized text denotes options available for selection under each category

CSS company-sponsored study, IIS investigator-initiated study, MAPP Medical Affairs Pharmaceutical Physicians

being involved in' (the lowest level of involvement) a given activity. Accountability was defined as having the power to veto an activity. Thus, if an individual is accountable for an activity and has the power to withhold this veto, then they personally underwrite the risk to the company of executing the activity. External stakeholders were regulators, payors, prescribers, and patients.

Participants used the ACRIN scale to rate the value of all internal stakeholders in each activity recorded in the retrospective study, and indicated which external stakeholders were influenced by each activity. Frequency counts of ACRIN classifications for each activity and the number of external stakeholders influenced by each activity were calculated from retrospective study data. The relative value of all internal stakeholders was measured to determine whether significant differences existed. A two-proportion $z$-test was used to test whether MAPPs were unique from other internal stakeholders in terms of being the sole professional rated as accountable for an activity that benefited at least one of the four external stakeholders. A one-tailed Mann-Whitney test was used to assess whether MAPPs were classified as being accountable for activities that benefited significantly more external stakeholders than other internal stakeholders if they were not accountable for activities that benefited all four stakeholders. A two-tailed Mann-Whitney test was used to assess whether MAPPs were classified as being 'consulted about', 'responsible for', or 'informed about' significantly more activities than other internal stakeholders. Data for the above tests were tabulated by their frequencies, and row percentage was calculated. Cross-tabulation determined the distribution of activity type across internal stakeholders. Pairwise comparisons between two internal stakeholders and between two activities were tested using a Chi-square test. All statistical analyses were performed using $\mathrm{R}$ version 4.0.2 (The R Foundation for Statistical Computing, Vienna, Austria), and $p$-values $<0.05$ were statistically significant.

\section{Results}

\subsection{Development of the MAPPval Instrument}

In total, 22 unique indicators were generated during the Awareness Round (1) of the Jandhyala method (Table 3). Payor-targeted activities and journal publications were the two highest-ranking metrics, with an awareness index of 1.00 and 0.98 , respectively. When the full list of indicators was presented to participants in the Consensus Round (2), a consensus index of 1.00 was observed for four of the indicators: multiple stakeholder-targeted (real-world evidence [RWE]) medical plans, protocols (investor-initiated or company-sponsored study), congress presentations, and accountable for activity. Overall, all indicators generated from the Awareness Round were retained in the final measure. 
Table 3 Twenty-two unique MAPP value indicators generated through the Jandhyala method

\begin{tabular}{llll}
\hline Codes & Statement & A & C \\
\hline 1 & Regulator-targeted activities (via regulatory affairs function) & 2 & 2 \\
2 & Payor-targeted activities (via market access function) & 1 & 2 \\
3 & Prescriber-targeted activities (via commercial and/or sales function) & 2 & 2 \\
4 & Patient-targeted activities (via patient advocacy function if available) & 2 & 2 \\
5 & Multiple-stakeholder-targeted (RWE) medical plan & 2 & 1 \\
6 & Submissions to external stakeholders & 2 & 2 \\
7 & Protocols (investigator-initiated study or company-sponsored study) & 2 & 1 \\
8 & Clinical study milestones & 2 & 2 \\
9 & Journal publications & 2 & 2 \\
10 & Congress posters & 2 & 2 \\
11 & Promotional approvals & 2 & 2 \\
12 & One-to-one meetings & 2 & 2 \\
13 & One-to-many meetings & 2 & 2 \\
14 & Advisory board (insight-generating) & 2 & 2 \\
15 & Congress presentations & 2 & 1 \\
16 & Training & 2 & 2 \\
17 & Responsible for activity & 2 & 2 \\
18 & Accountable for activity & 2 & 1 \\
19 & Consulted on activity & 2 & 2 \\
20 & Informed about activity & 2 & 2 \\
21 & Objective met & 2 & 2 \\
22 & Objective not met & 2 & 2 \\
\hline & & 2 & \\
\hline
\end{tabular}

$A$ awareness index, $C$ consensus index, $R W E$ real-world evidence, MAPP Medical Affairs Pharmaceutical Physicians

\subsection{Validation of the MAPPval Instrument}

The discriminant validity of the MAPPval instrument was assessed by implementing it in a retrospective study of MAPP job activities over a period of 12 months to determine whether it distinguished between the value of MAPPs and other stakeholders to pharmaceutical companies. In this study, MAPPs were classified as the only internal stakeholder accountable for at least one activity benefiting each of the four external stakeholders, and the difference between MAPPs and internal stakeholders on this measure of value was statistically significant $(p=0.039)$ (Table 4). Additionally, all internal stakeholders were classified as being accountable for at least one activity benefiting fewer than four external stakeholders (Table 5, Fig. 1). MAPPs were accountable for activities that influenced significantly more external stakeholders than the other internal stakeholders $(p<0.001)$. Finally, MAPPs were classified as accountable for $81 \%$ of the 229 activities recorded in the retrospective study (Table 6), while regulatory affairs, market access, and commercial stakeholders were classified as being collectively accountable for about $30 \%$ of the 229 activities (Fig. 2). Commercial stakeholders were classified as the stakeholder most informed about MAPP activities, at $37 \%$. Additionally, internal stakeholders were
Table 4 Number of internal stakeholders classified as accountable for at least one activity that benefited all four external stakeholders and fewer than four external stakeholders

\begin{tabular}{llll}
\hline $\begin{array}{l}\text { Internal stake- } \\
\text { holder }\end{array}$ & $\begin{array}{l}\text { All four external } \\
\text { stakeholders }(\%)\end{array}$ & $\begin{array}{l}\text { Fewer than four } \\
\text { external stakehold- } \\
\text { ers }(\%)\end{array}$ & $p$-value \\
\hline MAPP & $5(50)$ & $5(50)$ & - \\
Regulatory affairs & $0(0)$ & $10(100)$ & $0.039^{\mathrm{a}}$ \\
Market access & $0(0)$ & $10(100)$ & $0.039^{\mathrm{a}}$ \\
Commercial & $0(0)$ & $10(100)$ & $0.039^{\mathrm{a}}$ \\
Patient advocacy & $0(0)$ & $10(100)$ & $0.039^{\mathrm{a}}$ \\
\hline
\end{tabular}

MAPP Medical Affairs Pharmaceutical Physician

${ }^{a} \mathrm{~A} p$-value $<0.05$ was considered statistically significant using the two-proportion z-test

classified as not involved in a fairly large proportion of MAPP job activities: regulatory affairs $(51.53 \%)$, market access $(40.61 \%)$, commercial $(17.47 \%)$, and patient advocacy (66.81\%) (Table 6, Fig. 2). The total proportion of activities internal stakeholders were classified as 'consulted about', 'responsible for', and 'informed about' varied between MAPPs (18.34\%), regulatory affairs (44.10\%), market access (54.15\%), commercial (78.60\%), and patient advocacy $(32.75 \%)$. 
Table 5 Number of internal stakeholders classified as accountable for at least one activity that benefited $0,1,2$, or 3 external stakeholders

\begin{tabular}{llllllll}
\hline & $3(\%)$ & $2(\%)$ & $1(\%)$ & $0(\%)$ & Median & Mean & $p$-value \\
\hline MAPP & $4(80)$ & $1(20)$ & $0(0)$ & $0(0)$ & 3 & 2.8 & - \\
Regulatory affairs & $0(0)$ & $0(0)$ & $6(60)$ & $4(40)$ & 1 & 0.6 & $<0.001^{\text {a }}$ \\
Market access & $0(0)$ & $1(10)$ & $5(50)$ & $4(40)$ & 1 & 0.7 & $<0.001^{\text {a }}$ \\
Commercial & $0(0)$ & $2(20)$ & $2(20)$ & $6(60)$ & 0 & 0.6 & $<0.001^{\text {a }}$ \\
Patient advocacy & $0(0)$ & $0(0)$ & $1(10)$ & $9(90)$ & 0 & 0.1 & $<0.001^{\text {a }}$ \\
\hline
\end{tabular}

MAPP Medical Affairs Pharmaceutical Physician

${ }^{\mathrm{a}} \mathrm{A} p$-value $<0.05$ was considered statistically significant using the Mann-Whitney test

\section{Discussion}

The current lack of a measure for MAPP performance in the pharmaceutical industry as well as their potential undervaluation in the pharmaceutical industry and vulnerability to role replacement (Jandhyala R, MBBS, unpublished data, 2020) necessitated the development and validation of the MAPPval instrument. While MAPPs have been recognized as playing an important role in the pharmaceutical industry in terms of ensuring the predominance of scientific integrity over commercial interests and the transition of drugs from R\&D to commercial $[25,26]$, their role and value have not been formally characterized. Conceptualizing and operationalizing a new construct such as the MAPPval requires careful selection of indicators to ensure complete accuracy. Neutral theory describes the need for the generation of an exhaustive list of relevant indicators, being careful to exclude any irrelevant information from its observation [27, Jandhyala (2020, unpublished data)]. Neutral theory has been successfully operationalized using the Jandhyala method in the development of similar instruments for the measurement of diseasespecific quality of life [23] and disease severity of a rare disease [24]. The MAPPval instrument was generated by the consensus of 10 MAPPs with at least 2 years of experience in UK pharmaceutical companies and contained 22 unique indicators of MAPP value to pharmaceutical companies. The discriminant validity of the MAPPval instrument was shown in a retrospective study by answering the following research questions. (1) Is the MAPP the only internal stakeholder who has been classified as accountable for at least one activity benefiting each of the four external stakeholders? (2) Has the MAPP been classified as accountable for activities benefiting significantly more external stakeholders than each of the other internal stakeholders? (3) Is the number of times the MAPP has been classified as 'consulted about', 'responsible for' or 'informed about' significantly greater than each of the other internal stakeholders?

First, the MAPP was the only internal stakeholder classified as accountable for at least one activity involving each of the four external stakeholders. MAPPs partner with pharmacists, access providers, diagnostic groups, insurance providers, patients and patient advocacy groups, technology companies, and entrepreneurs, as well as the general community, to enhance patient-centric healthcare [28]. Thus, influencing external stakeholders through their job activities is key to their role, which was reflected in the findings of this study. Medicine adoption, the ultimate goal of pharmaceutical companies, requires successful engagements with the four stakeholders (regulators, payors, prescribers, and patients) $[29,30]$, therefore MAPPs can be seen as having unique value to pharmaceutical companies as the only internal stakeholder accountable for activities engaging with all four stakeholders. As employee performance has been defined as 'the behaviors and actions that support organizational goals' [31], the MAPPval instrument can also be said to accurately reflect the performance of MAPPs in this respect. MAPPs play a critical role in guaranteeing that key messages are informed by sound scientific evidence and no disingenuous assertions are made [32], which explains their high level of accountability for pharmaceutical company activities. MAPPs also design and implement RWE to answer questions unanswered by registered clinical trials $[33,34]$, therefore their value is not only in their ability to influence successful medicine adoption but to do so in many different ways. Thus, the findings of this study suggested that MAPPs were of key value to pharmaceutical company success and they were the only internal stakeholder of those studied able to deliver this value.

Second, MAPPs were classified as accountable for activities targeting significantly more external stakeholders than other internal stakeholders, even when their activities did not influence all four external stakeholders. Observations within this study suggested some variation between MAPPs regarding the overall degree of accountability they held, which may explain why some MAPPs were accountable only for activities that influenced fewer than all four stakeholders. This may have been driven by variation between the scope of MAPP job roles, for example if a regulatory affairs role was being fulfilled by a pharmaceutical physician or if the MAPP reported into a more senior MAPP in the organization, who carried the accountability for certain activities. These MAPPs were observed to be accountable for activities that targeted three and two external stakeholders, respectively. Despite this, the comparative value of the 

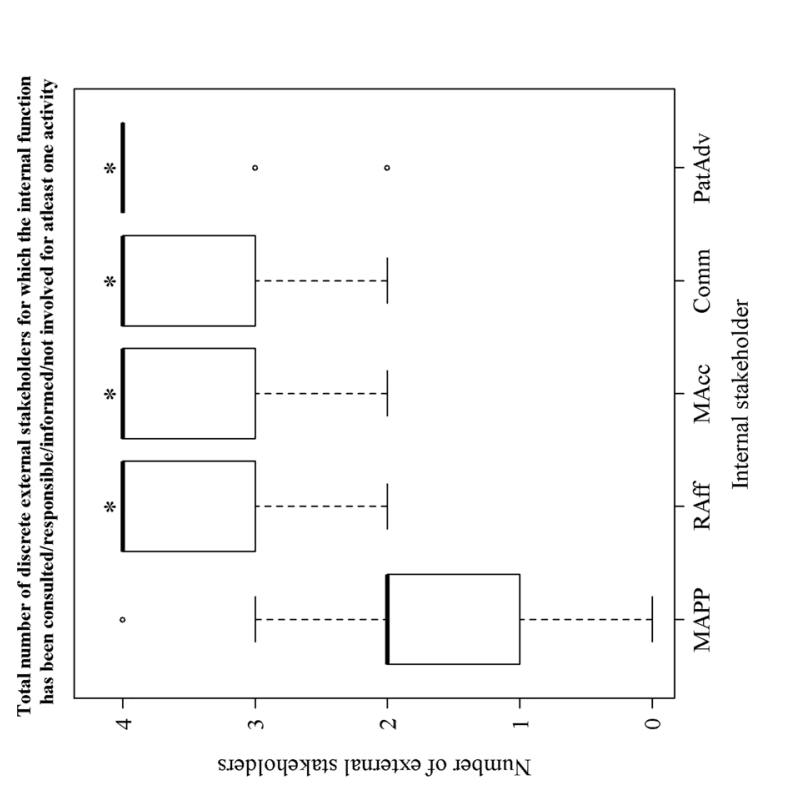

ठे

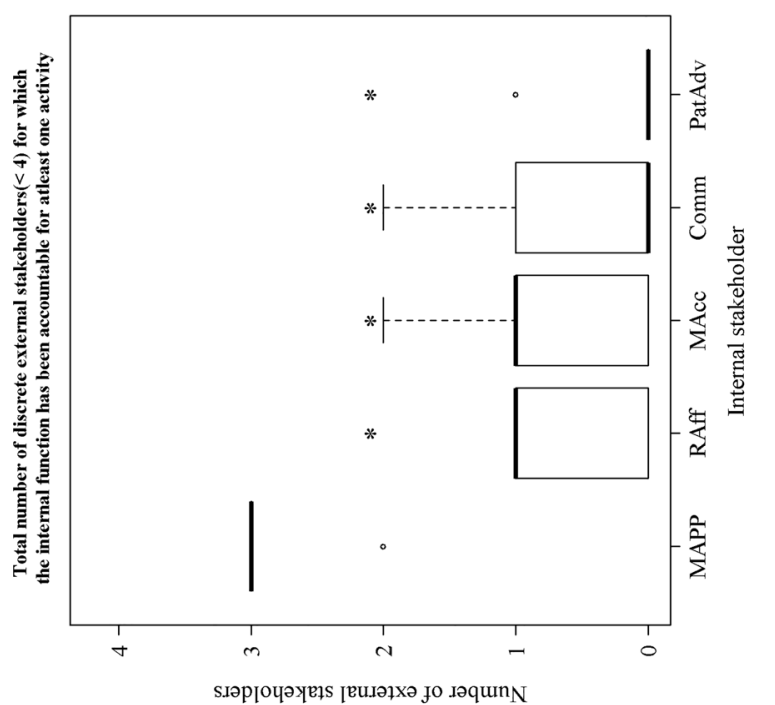

के

응

焉 $\frac{\omega}{0}$

สี పี

这苛

4 可

岂氞

苛㟃

華通

承

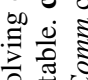

哥

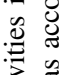

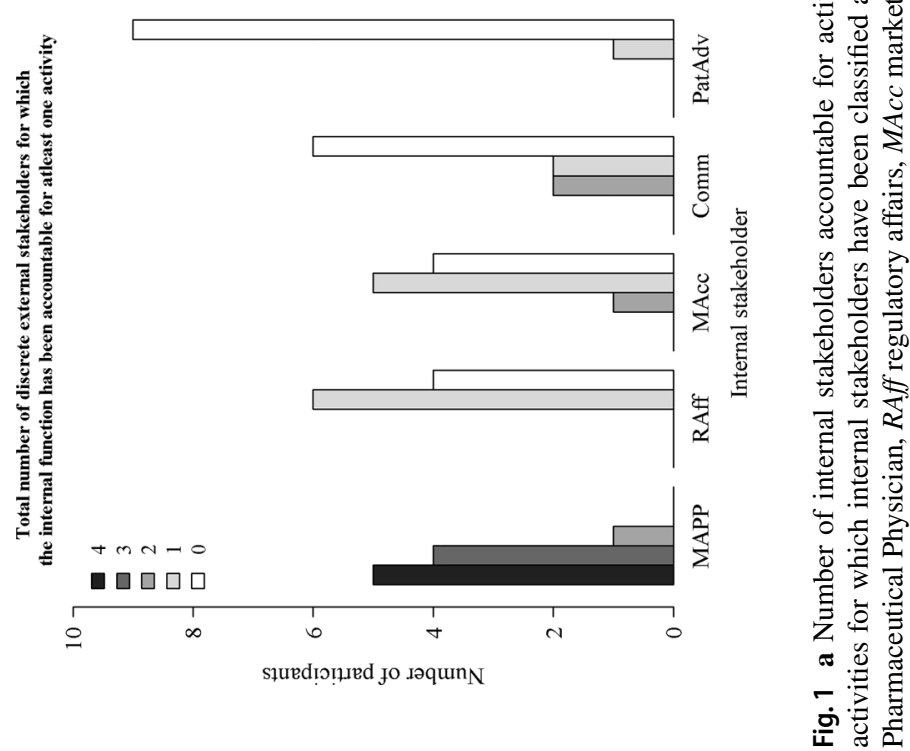


Table 6 Internal stakeholder value for activities recorded in the retrospective study
Fig. 2 Accountability of internal stakeholders for all 229 MAPP activities identified. MAPP Medical Affairs Pharmaceutical Physician

\begin{tabular}{lllllll}
\hline & \multicolumn{2}{l}{ Number $(\%)$ of activities } & & & \\
\cline { 2 - 6 } & Accountable & Consulted & Responsible & Informed & Not involved & Total \\
\hline MAPP & $187(82)$ & $22(10)$ & $15(7)$ & $5(2)$ & $0(0)$ & 229 \\
Regulatory affairs & $10(4)$ & $73(32)$ & $8(3)$ & $20(9)$ & $118(52)$ & \\
Market access & $12(5)$ & $70(31)$ & $8(3)$ & $46(20)$ & $93(41)$ & \\
Commercial & $9(4)$ & $69(30)$ & $26(11)$ & $85(37)$ & $40(17)$ & \\
Patient advocacy & $1(0.4)$ & $32(14)$ & $5(2)$ & $38(17)$ & $153(67)$ & \\
\hline
\end{tabular}

Using the Mann-Whitney (two-tailed) test

MAPP Medical Affairs Pharmaceutical Physician

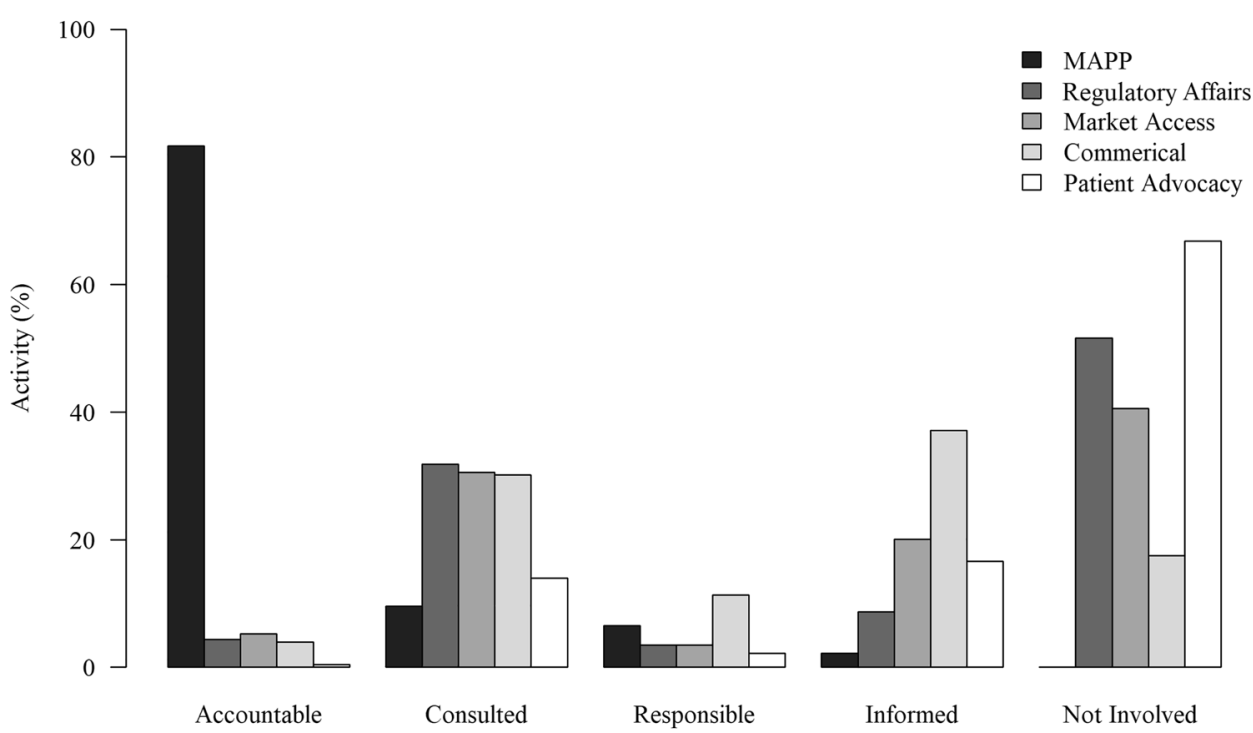

MAPP function in terms of its influence of external stakeholders was higher than that of other internal stakeholders to a statistically significant degree (Fig. 1). Additionally, the findings of this study reflected the role responsibilities of stakeholders other than MAPPs. Commercial stakeholders were the most 'consulted' (30.13\%) and 'informed about' (37.12\%) activities, and, apart from MAPPs, the least 'not informed' (17.4\%) about activities. This reflected the nature of their role within pharmaceutical companies, as commercial departments are almost exclusively led by program facilitators who must meet commercial targets by coordinating projects involving many different internal stakeholders. Patient advocacy was 'not involved in' most (66.81\%) activities. This was expected, as this role is emergent and currently has a narrow scope. The patient is the ultimate beneficiary of pharmaceutical company activities and there is an ever-increasing emphasis on their inclusion as a stakeholder, therefore patient advocacy may become more involved in activities over time.

Third, MAPPs were classified as 'consulted about', 'responsible for', or 'informed about' fewer times than other internal stakeholders. This was because the MAPP was almost always classified as accountable for activities in which they were involved. Of the total 229 activities performed, MAPPs were classified as accountable for 187 (81.7\%). Consequently, they showed value at every level: accountable, consulted, responsible, and informed, as described in Table 6. The MAPP has traditionally been considered a role that supports other stakeholders within pharmaceutical companies [2]; however, the results of this study were more in line with the idea that MAPPs lead the pharmaceutical company activities that influence external stakeholders and are supported in these activities by other stakeholders [2]. For regulatory affairs, market access, and commercial stakeholders, this support was in the form of consulting on $31.88 \%, 30.57 \%$, and $30.13 \%$ of activities, respectively (Table 6). Additionally, internal stakeholders were classified as not involved in a fairly large proportion of MAPP job activities: regulatory affairs (51.53\%), market access $(40.61 \%)$, commercial (17.47\%), and patient advocacy $(66.81 \%)$. The relatively narrow scope of the role of these internal stakeholders in comparison with MAPPs was 
reflected by the fact that they were classified as accountable for activities that only generally benefited one or two external stakeholders, while half the MAPPs were accountable for at least one activity that benefited all four external stakeholders. The centrality of the four external stakeholders to pharmaceutical company success supports the unique value of MAPPs in terms of their sole accountability for activities that benefit a higher number of stakeholders than regulatory affairs, market access, commercial, and patient advocacy.

\subsection{Outlook and Future Work}

The MAPPval instrument can be used to measure MAPP performance within pharmaceutical companies to assess and enhance the development of MAPPs and demonstrate their value as well as providing a benchmark against which to standardize and develop the utility of MAPPs to stakeholders in the pharmaceutical industry in the UK. Work performance measures are commonly used in performance management and organizational decision-making [35], and the MAPPval instrument could be applied to these purposes. It may be useful to explore the construct validity of the measure with a larger sample of MAPPs and to adapt the measure to other geographical contexts, especially those in which the development of the MAPP role within pharmaceutical companies may enhance industry practices and accountability. This tool has demonstrated the need to view MAPPs as valuable and unique members of the multidisciplinary team within pharmaceutical companies, especially in terms of their accountability for vital activities that benefit all external stakeholders.

\subsection{Study Limitations}

The study was limited, as it considered the MAPP role and pharmaceutical company activities only from the MAPP's perspective. Thus, the focus was on intrinsic rather than extrinsic value. While this limits the scope of the measure to applications that utilize measures of intrinsic value, it does not necessarily affect its validity, and intrinsic measures are commonly used in work performance measurement [35]. Furthermore, MAPPs fulfil a regulatory role within pharmaceutical companies in respect of engagements with external stakeholders, therefore they are likely to be involved in virtually all pharmaceutical company activities involving external stakeholders. As pharmaceutical company success is dependent on successful engagements with external stakeholders, it can be argued that the scale is valid in this respect. Further research could develop the measure to include extrinsic parameters, widening its potential application. Additionally, it was possible that the retrospective study was subject to recall bias, which may lead to between-participant variation in quality and quantity of data. However, all
MAPPs who completed the retrospective study participated in the first phase of the study, which involved a Consensus Round that standardized awareness of MAPP professional activities between participants, mitigating this concern to some extent. Additionally, while all participants had at least 2 years of experience within a UK pharmaceutical company at a regional or global level, detailed information on their career stage was not collected, and this may have influenced the scope of their role and their perceptions of it. Finally, the study was conducted in a UK context, therefore the results cannot be said to be generalizable to other countries, which may vary in terms of pharmaceutical company engagement with external stakeholders and professional regulation of MAPPs.

\section{Conclusions}

The MAPPval instrument contained 22 unique indicators and was validated by a retrospective study completed by MAPPs. The MAPPval instrument differentiated MAPPs from other internal stakeholders, as they were viewed as the only one accountable for at least one activity that influenced all external stakeholders and were accountable for activities that targeted significantly more external stakeholders than other internal stakeholders. They were also found to be accountable for significantly more job activities in which they were involved than other internal stakeholders. Thus, their level of value to the company was higher than that of other internal stakeholders. MAPPs were the only internal stakeholder accountable for activities targeting all gatekeeper stakeholders involved in medicine adoption, therefore their value to pharmaceutical companies is unique. The MAPPval instrument has defined the MAPP role and can be used to assess MAPP job performance and standardize the quality of MAPP work in the pharmaceutical industry, and can be developed by further research.

Supplementary Information The online version contains supplementary material available at https://doi.org/10.1007/s40290-021-00413-9.

Acknowledgements The author would like to acknowledge the contribution of Medialis personnel (Mohammed Kabiri, Omolade FemiAjao, Lauri Naylor, and Brendon Pearce) in the development of the MAPPval instrument and the writing of this manuscript, as well as the MAPP advisory panel (Dr Andy Pain, Dr Lisa Moore-Ramdin, Dr William Spencer, Dr Daniel Franks, Dr Gerd Möller, Dr John Bolodeoku, Dr Daniel A. Thomas, Dr Michael Smyth, Dr Arun Mistry, Dr Timir Patel, and Dr Raj Rout) for their participation in this study.

\section{Declarations}

Funding The author received no funding for this particular work. 
Competing interests Ravi Jandhyala is a visiting senior lecturer at the Centre for Pharmaceutical Medicine Research at King's College London, and is responsible for research into RWE approaches. He is also the Founder and CEO of Medialis Ltd, a medical affairs consultancy and contract research organization involved in the design and delivery of RWE, including patient-reported outcomes and patient registries.

Ethical approval The study was approved by the King's College Research Ethics Committee (reference number: MRA-20/21-22441).

Consent to participate All study participants provided written consent to participate in this study.

\section{Consent for publication Not applicable.}

Transparency Medialis Ltd continues to support the Medical Affairs Pharmaceutical as part of its Corporate Social Responsibility Project.

Availability of data and material Data from this study will be made available upon reasonable request to the author.

Code availability Not applicable.

Author contributions RJ conducted the study and prepared, authored, and approved the manuscript. He also designed and implemented the MAPPval instrument. He affirms that the manuscript is an honest, accurate, and transparent account of the study being reported, that no important aspects of the study have been omitted, and any discrepancies from the study as planned (and, if relevant, registered) have been explained.

Open Access This article is licensed under a Creative Commons Attribution-NonCommercial 4.0 International License, which permits any non-commercial use, sharing, adaptation, distribution and reproduction in any medium or format, as long as you give appropriate credit to the original author(s) and the source, provide a link to the Creative Commons licence, and indicate if changes were made. The images or other third party material in this article are included in the article's Creative Commons licence, unless indicated otherwise in a credit line to the material. If material is not included in the article's Creative Commons licence and your intended use is not permitted by statutory regulation or exceeds the permitted use, you will need to obtain permission directly from the copyright holder. To view a copy of this licence, visit http://creativecommons.org/licenses/by-nc/4.0/.

\section{References}

1. Stonier PD, Silva H, Lahon H. Pharmaceutical medicine. Int J Pharm Med. 2007;21(4):253-62.

2. Setia $\mathrm{S}$, et al. Evolving role of pharmaceutical physicians in medical evidence and education. Adv Med Educ Pract. 2018;9:777-90.

3. Fda US. The drug development process. US FDA; 2018.

4. British Medical Association. The pharmaceutical physician. British Medical Association; 2013.

5. Khosla S, et al. Real world evidence (RWE) - a disruptive innovation or the quiet evolution of medical evidence generation? F1000Research. 2018;7(111):1-14.

6. Sweiti $\mathrm{H}$, et al. Physicians in the pharmaceutical industry: their roles, motivations, and perspectives. Drug Discov Today. 2019;24(9):1865-70.

7. Green BN, Johnson CD. Interprofessional collaboration in research, education, and clinical practice: working together for a better future. J Chiropr Educ. 2015;29(1):1-10.
8. Jackson SHD, Mangoni AA, Batty GM. Optimization of drug prescribing. Br J Clin Pharmacol. 2004;57(3):231-6.

9. Grol R. Successes and failures in the implementation of evidencebased guidelines for clinical practice. Med Care. 2001;39(8 Suppl 2):46-54.

10. Cochrane LJ, Olson CA, Murray S, Dupuis M, Tooman T, Hayes S. Gaps between knowing and doing: understanding and assessing the barriers to optimal health care. J Contin Educ Health Prof. 2007;27(2):94-102.

11. Buchan H. Gaps between best evidence and practice: causes for concern. Med J Aust. 2004;180(6 Suppl):48.

12. Setia S, Subramaniam K, Tay JC, Teo BW. Hypertension and blood pressure variability management practices among physicians in Singapore. Vasc Health Risk Manag. 2017;13:275-85.

13. Setia S, Subramaniam K, Teo BW, Tay JC. Ambulatory and home blood pressure monitoring: gaps between clinical guidelines and clinical practice in Singapore. Int J Gen Med. 2017;10:189-97.

14. Setia S, Fung SS, Waters DD. Doctors' knowledge, attitudes, and compliance with 2013 ACC/AHA guidelines for prevention of ath-erosclerotic cardiovascular disease in Singapore. Vasc Health Risk Manag. 2015;11:303-10.

15. Saslow D, Solomon D, Lawson HW, et al. American Cancer Society, American Society for Colposcopy and Cervical Pathology, and American Society for Clinical Pathology screening guidelines for the prevention and early detection of cervical cancer. CA Cancer J Clin. 2012;62(3):147-72.

16. Komajda M, Lapuerta P, Hermans N, et al. Adherence to guidelines is a predictor of outcome in chronic heart failure: the MAHLER survey. Eur Heart J. 2005;26(16):1653-9.

17. Mccullough ML, Patel AV, Kushi LH, et al. Following cancer prevention guidelines reduces risk of cancer, cardiovascular disease, and all-cause mortality. Cancer Epidemiol Biomark Prev. 2011;20(6):1089-97.

18. Jandhyala R. Influence of pharmaceutical company engagement activities on the decision to prescribe: a pilot survey of UK Rare Disease Medicine Prescribers. Pharmaceut Med. 2020;34(2):127-34.

19. Fickweiler F, Fickweiler W, Urbach E. Interactions between physicians and the pharmaceutical industry generally and sales representatives specifically and their association with physicians' attitudes and prescribing habits: a systematic review. BMJ Open. 2017;7(9):e016408.

20. Silva $\mathrm{H}$, et al. Core competencies for pharmaceutical physicians and drug development scientists. Front Pharmacol. 2013;4:105.

21. Jandhyala R. A novel method for observing proportional group awareness and consensus of items arising from list-generating questioning. Curr Med Res Opin. 2020;36(5):883-93.

22. Jandhyala R. Delphi, non-RAND modified Delphi, RAND/UCLA appropriateness method and a novel group awareness and consensus methodology for consensus measurement: a systematic literature review. Curr Med Res Opin. 2020;36(11):1873-87. https:// doi.org/10.1080/03007995.2020.1816946.

23. Jandhyala R. PAC-19QoL: design, validation and implementation of the post-acute (long) COVID-19 quality of life (PAC-19QoL) instrument. Health Qual Life Outcomes. 2021;19(1):229. https:// doi.org/10.1186/s12955-021-01862-1.

24. Damy T, Conceição I, García-Pavía P, Gillmore J, Jandhyala R, Sabbat J, et al. A simple core dataset and disease severity score for hereditary transthyretin (ATTRv) amyloidosis. Amyloid. 2021;28(3):189-98. https://doi.org/10.1080/13506129.2021. 1931099.

25. Jain S. Bridging the Gap Between R\&D and commercialization in pharmaceutical industry: role of medical affairs and medical communications. Int J Clin Biomed Res. 2017;3:44-9. 
26. Grom T. Medical affairs: beyond the science. Showcase feature. 2013. http://www.pharmavoice.com/article/medical-affairsbeyond-the-science/. Accessed 8 September 2021.

27. Jandhyala R. Neutral theory: applicability and neutrality of using generic health-related quality of life tools in diseases or conditions where specific tools are available. BMC Med Res Methodol. 2021;21(1):86. https://doi.org/10.1186/s12874-021-01279-w.

28. Rollins BL, Perri M. Pharmaceutical Marketing. Burlington: Jones \& Bartlett Learning; 2014.

29. Verbaanderd C, Rooman I, Meheus L, Huys I. On-Label or Offlabel? overcoming regulatory and financial barriers to bring repurposed medicines to cancer patients. Front Pharmacol. 2020;10:1664. https://doi.org/10.3389/fphar.2019.01664.

30. Keene ON, Ruberg S, Schacht A, et al. What matters most? Different stakeholder perspectives on estimands for an invented case study in COPD. Pharm Stat. 2020;19(4):370-87. https://doi.org/ 10.1002/pst.1986.

31. Campbell JP. Modeling the performance prediction problem in industrial and organizational psychology. In: Dunnette MD,
Hough LM, editors. Handbook of industrial and organizational psychology. Palo Alto: Consulting Psychologists Press; 1990. p. 687-732.

32. Plantevin L, Schlegel C, Gordian M. Reinventing the Role of Medical Affairs. 2017. http://www.bain.com/publications/artic les/reinventing-the-role-of-medical-affairs.aspx. Accessed $8 \mathrm{Sep}$ 2021.

33. Jandhyala R. The multiple stakeholder approach to real-world evidence (RWE) generation: observing multidisciplinary expert consensus on quality indicators of rare disease patient registries (RDRs). Curr Med Res Opin. 2021;37(7):1249-57.

34. Jandhyala R. A medicine adoption model for assessing the expected effects of additional real-world evidence (RWE) at product launch. Curr Med Res Opin. 2021;37(9):1645-55.

35. van der Vaart L. The performance measurement conundrum: Construct validity of the Individual Work Performance Questionnaire in South Africa. S Afr J Econ Manag Sci. 2021;24(1):3581. https://doi.org/10.4102/sajems.v24i1.3581. 